\title{
NEAR: new earths in the Alpha Cen Region (bringing VISIR as a "visiting instrument" to ESO-VLT-UT4)
}

Hans-Ulrich Käufl, Markus Kasper, Robin Arsenault, Gerd Jakob, Serban Leveratto, et al.

Hans-Ulrich Käufl, Markus Kasper, Robin Arsenault, Gerd Jakob, Serban Leveratto, Gerard Zins, Eloy Fuenteseca, Miguel Riquelme, Ralf Siebenmorgen, Michael Sterzik, Nancy Ageorges, Sven Gutruf, Dirk Kampf, Arnd Reutlinger, Olivier Absil, Brunella Carlomagno, Olivier Guyon, Pete Klupar, Dimitri Mawet, Garreth Ruane, Mikael Karlsson, Eric Pantin, Kjetil Dohlen, "NEAR: new earths in the Alpha Cen Region (bringing VISIR as a " visiting instrument" to ESO-VLT-UT4)," Proc. SPIE 10702, Ground-based and Airborne Instrumentation for Astronomy VII, $107020 D$ (6 July 2018); doi: 10.1117/12.2313395

Event: SPIE Astronomical Telescopes + Instrumentation, 2018, Austin, Texas, United States 


\title{
NEAR: New Earths in the Alpha Cen Region (bringing VISIR as a "visiting instrument" to ESO-VLT-UT4)
}

\author{
Hans Ulrich Käuf ${ }^{a}$ Markus Kasper ${ }^{a}$ Robin Arsenaut $^{a}$ Gerd Jakob $^{a}$ Serban Leveratto $^{a b}$ \\ Gerard Zins ${ }^{c}$ Eloy Fuenteseca ${ }^{c}$ Miguel Riquelme ${ }^{c}$ Ralf Siebenmorgen ${ }^{a}$ Michael Sterzik $^{a}$ \\ Nancy Ageorges ${ }^{d}$ Sven Gutruf $^{d}$ Dirk Kampf $^{d}$ Arnd Reutlinger $^{d}$ Olivier Absil ${ }^{e}$ \\ Brunella Carlomagno ${ }^{e}$ Olivier Guyon ${ }^{f}$ Pete Klupar $^{g}$ Dimitri Mawet ${ }^{h i}$ Garreth Ruane $^{h}$ \\ Mikael Karlsson ${ }^{j}$ Eric Pantin $^{k}$ Kjetil Dohlen $^{l}$ \\ ${ }^{a}$ European Southern Observatory, Karl Schwarzschild Strasse 2, \\ D-85746 Garching bei München, Germany; \\ ${ }^{b}$ TU Delft, Postbus 5, 2600 AA Delft, The Netherlands;
}

${ }^{c}$ European Southern Observatory, Alonso de Cordoba 3107, Vitacura, 7630355 Santiago, Chile;

${ }^{d}$ Kampf Teleskop Optik, Alois-Gilg-Weg 7, D-81373 München, Germany;

eSpace Sciences, Technologies \& Astrophysics Research (STAR) Institute, Université de Liège,

Bat B5c, 4000 Sart Tilman, Belgium;

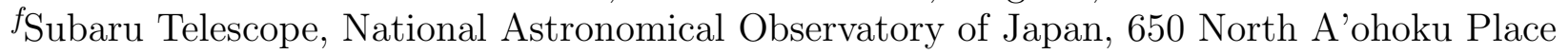

Hilo, HI 96720, U.S.A.;

${ }^{g}$ Breakthrough Initiatives, 3000 Sand Hill Road, 4-180, Menlo Park, CA 94025, U.S.A.;

${ }^{h}$ Department of Astronomy, MC 249-17 Caltech, 1200 E. California Blvd.,

Pasadena, CA 91125, U.S.A.;

${ }^{i}$ Jet Propulsion Laboratory, California Institute of Technology

Pasadena, CA 91109, U.S.A.;

$j$ Ångström Laboratory, P.O Box 516, 751 20, Uppsala, Sweden;

${ }^{k}$ CEA Saclay, Orme des Merisiers Bat. 709, 91191 Gif sur Yvette Cedex, France;

${ }^{l}$ Laboratoire d'Astrophysique de Marseille (LAM), 38 rue Frédéric Joliot-Curie,

13388 Marseille Cedex 13, France;

\begin{abstract}
ESO in collaboration with the Breakthrough Initiatives, is adding a dedicated coronagraph to the Very Large Telescope mid-IR imager (VISIR) to further boost the high dynamic range imaging capability of this instrument.

After the VISIR upgrade in 2012, where coronagraphic masks were first added to VISIR, it became evident that coronagraphy at a ground-based $8 \mathrm{~m}$-class telescope, even at wavelengths as long as $10 \mu \mathrm{m}$, critically needs adaptive optics. For VISIR, a work-horse observatory facility instrument in normal operations, this is "easiest" achieved by bringing VISIR as a visiting instrument to the ESO-VLT-UT4 having an adaptive M2.

This "visit" enables a meaningful search for Earth-like planets in the habitable zone around both $\alpha$-Cen 1 and $\alpha$-Cen $n_{2}$. Meaningful here means, achieving a contrast of $\approx 10^{-6}$ within $\approx 0.8$ arcsec from the star. Various measures to improve the sensitivity of VISIR will be applied, especially a dedicated filter, faster chopping and a Strehl-ratio close to $100 \%$ thanks to extreme adaptive optics. This should allow to detect a planet twice the diameter of Earth in $50 \mathrm{~h}$ on source integration time. Key components will be a diffractive coronagraphic mask, the annular groove phase mask (AGPM), optimized for the most sensitive spectral band-pass in the N-band, complemented by a sophisticated apodizer at the level of the Lyot stop. For VISIR noise filtering based on
\end{abstract}

Further author information: (Send correspondence to H.U.K.)

H.U.K.: E-mail: hukaufl@eso.org, Telephone: +49 893206414

M.K..: E-mail: mkasper@eso.org, Telephone: +49 32006359

Ground-based and Airborne Instrumentation for Astronomy VII, edited by Christopher J. Evans, Luc Simard, Hideki Takami, Proc. of SPIE Vol. 10702, 107020D · (C) 2018 SPIE · CCC code: 0277-786X/18/\$18 · doi: 10.1117/12.2313395 
fast chopping is required. A novel internal chopper system will be integrated into the cryostat. This chopper is based on the standard technique from early radio astronomy, conceived by the microwave pioneer Robert Dicke in 1946, which was instrumental for the discovery of the 3K microwave background.

For risk mitigation all components are being tested and qualified under realistic conditions in the lab at ESO headquarters before integration into the instrument. The performance or suppression of the coronagraph is so good, that a non-thermal source (vulgo a laser) is needed on the test-bench.

We will give an overview of the optical changes to VISIR, the implementation of wave front sensing, the Dicke switch design and laboratory testing, the AGPM design and laboratory testing, non common path error control with a ZELDA mask, sensitivity and contrast estimates, data flow and analysis, the overall project status, plan and outlook

Needless to say that this project is of critical interest for future infrared instrumentation at the next generation of extremely large telescopes aiming at surveying the solar neighborhood for terrestrial planets by detecting and characterizing them based on their mid-IR fluxes.

Keywords: exo-Earth, alpha-Cen, habitable zone, coronagraphy, apodization, extreme AO, thermal infrared

\section{INTRODUCTION}

At ESO there is an almost un-interrupted history of facility class state-of-the-art instrumentation for observation in the extreme thermal infrared, i.e. at wavelengths from 8 to $24 \mu m$ dating back to $1980 .{ }^{1}$ The last two instruments in this row, TIMMI2 $2^{2,3}$ and VISIR $^{4}$ are instrumental for the NEAR project. TIMMI2 has been retired from ESO's 3.6m telescope in 2007 and serves as detector test facility at ESO's headquarters in Garching. Extended by an optical bench TIMMI2 also serves as test facility for all hardware which is needed for a successful transformation of the facility instrument VISIR into an extreme-AO high contrast imager.

VISIR, first commissioned in 2004 underwent a major upgrade ${ }^{5,6}$ in 2012. This upgrade encompassed the replacement of the detector and adding of a prism to the grating turret as a low resolution spectrograph. As it was very little extra effort, the team also added Sparse Aperture Masking and coronagraphy as special observing modes for bright and compact objects. The implementation of coronagraphy was based on two technologies: 4-quadrant phase masks ${ }^{7}$ and a then novel and revolutionary annular groove phase mask..$^{8,9}$ Details of the specific device in use in VISIR since 2012 have been published elsewhere. ${ }^{10}$ All these modes could be successfully commissioned and tested on sky ${ }^{11}$ but it became immediately clear that the application of coronagraphy was limited by the instability of the PSF at the VLT.

During the upgrade of VISIR, in parallel and unrelated, the ESO Very Large Telescope Unit Telescope number 4 (VLT-UT4) was being transformed into the Adaptive Optics Facility (AOF). The original secondary mirror of this telescope has been replaced with an adaptive secondary mirror featuring 1172 fast actuators. ${ }^{12}$ Given that at $\lambda \approx 10 \mu \mathrm{m}$ the Fried parameter $R_{0}$ is of order of $6-9 \mathrm{~m}$ while the atmospheric coherence time is of order of $300 \mathrm{~ms}^{13}$ the AOF features truly extreme adaptive optics here. Compared to "normal" post-focal plane AO-systems the AOF does not add any additional warm optical surfaces to the scientific light path. Hence there is no increase in thermal background compared to classical non-AO operations. VISIR at UT3 without AO, on the other hand, was always plagued with insufficient image quality delivered by the telescope. TIMMI2 at the $3.6 \mathrm{~m}$ telescope $(\lambda / D \approx 0.7 \mu \mathrm{m}$ at $\lambda=10 \mu \mathrm{m})$ showed an extremely stable PSF, almost at the diffraction limit. ${ }^{14}$ Indeed, it came as a surprise that the change of telescope diameter by a factor of two resulted in a "boiling" PSF, even though techniques like fast $(\approx 100 \mathrm{~Hz}$ bandwidth) tip-tilt correction were applied. On the other hand, the transformation of the VLT-UT4 into an Adaptive Optics integrated telescope proceeded extremely well. Hence the idea developed to move VISIR from its "home", the Cassegrain Focus of UT3 to the Cassegrain focus of UT4 for a deep coronagraphic imaging campaign of the closest 'normal' stars next to Earth.

\section{SELECTION OF SEARCH WAVELENGTH AND SENSITIVITY}

It is a well known fact that the contrast for detection of Earthlike planets around stars of Solar type is most favorable in the mid-infrared. Still, given the well known dramatic drop in sensitivity for ground based instru- 
ments in the thermal IR due to the strong background radiation, ${ }^{*}$ it is not obvious whether the flux from the planet will exceed the noise limits of instruments like VISIR.

The diffraction limit tends to favour shorter wavelengths. On the other hand scattering scales with $\lambda^{-1.3}-\lambda^{-4}$ and the intensitity of residual speckles also drops with $\lambda^{-2}$. In total, extremely high contrast imaging seems to be 'easier' at longer wavelengths.

While the detection of Earth at $\lambda \approx 10 \mu m$ in the habitable zones around $\alpha-C e n_{1}$ and $\alpha-C e n_{2}$ will be relatively easy with future extremely large telescopes ${ }^{13}$ the feasibility of a detection with today's $8 \mathrm{~m}$-class telescopes is more challenging and needs some further analysis.

Data from an ongoing VISIR program (098.C-0050, Principal Investigator M. Sterzik) was used to measure the actual point source sensitivity and estimate the expected background-limited imaging performance (BLIP) of NEAR. The data consist of nine one-hour observations of Sirius recorded between December 2016 and March 2017 in the B10.7 $(10.2 \leq \lambda \leq 10.2 \mu m)$ filter with $4-\mathrm{Hz}$ chopping.

We measured the total flux of Sirius and used it to scale the VLT's Airy pattern. This is a reasonable assumption given the nearly perfect AO-corrected PSF in the N-band. The background noise per pixel was also measured. The resulting SNR was derived for different photometric aperture diameters. An aperture of $1.25 \lambda / D$ diameter, containing $60 \%$ of the total flux spread over 50 pixels, is optimum and provides an average BLIP sensitivity of $1.1 \mathrm{mJy}$ ( $5 \sigma$ in $1 \mathrm{~h}$ elapsed time). In BLIP the resulting noise is dominated by the shot-noise of the huge back ground flux. Thus the oversampling of the PSF does not compromise point source sensitivity.

Scaling the SNR of the classical VISIR data to the new NEAR configuration considering the various changes in the setup (spectral filter $10.0 \leq \lambda \leq 12.0 \mu \mathrm{m}$, AGPM, Lyot stop, AO correction, dichroic, etc) yields the expected close to BLIP sensitivity of $0.7 \mathrm{mJy}$ ( $5 \sigma$ in $1 \mathrm{~h}$ elapsed time).

Last but not least, it was also verified that in VISIR S/N scales with $\sqrt{t_{o b s}}$ for long integration times and stacking of multiple exposures. The analysis shows that the noise is indeed spatially and temporally uncorrelated (the noise covariance matrix between the nine data sets is almost diagonal), and noise statistics scales with integration time as expected. Therefore, we expect a final sensitivity of the 100-hr NEAR campaign of $70 \mu J y$, which is sufficient to detect a 1.9-Earth-radii planet with an Earth-like emission spectrum or a 1.3-Earth-radii desert planet emitting like a $325 \mathrm{~K}$ black body the detection of a planet, twice the diameter of Earth in the habitable zone of both components of $\alpha-C e n$.

\section{DETAILS ON THE IMPLEMENTATION OF CORONAGRAPHY}

\subsection{The AGPM Coronagraph}

The VISIR imager optics features a cryogenic image plane, well protected by a set of cold baffles. Then a reflective collimator forms a pupil with $\approx 18 \mathrm{~mm}$ diameter in a well collimated beam. At this position (c.f. figure 1) there is a filter wheel with 40 positions. At this location, Lyot stops, apodizers etc can conveniently be placed. Thereafter there is a re-imager by means of a three-mirror-anastigmat (TMA). In the VISIR detector plane this yields a scale of 45 mas per pixel $(30 \mu m$ pitch on a $1 k \times 1 k$ Raytheon Aquarius As:Si BIB detector) to be compared with the diffraction limit $(1.22 \lambda / D=310 \mathrm{mas})$. Under these conditions - as pointed out above - oversampling does not introduce additional noise. Hence a relatively luxurious sampling is possible, which also helps to reject detector artifacts. VISIR is mounted to the VLT-Cassegrain adaptor via a rotator allowing to freely rotate the instrument. This allows to rotate the instrument pupil of VISIR and its associated masks in order to keep it well aligned with the telescope pupil. As the VISIR detector is being read with individual integration times of order of $10 \mathrm{~ms}$ the associated field rotation can be easily compensated in the data reduction software. On the other hand, quasi static speckles which interfere with the detection of faint objects close to bright stars are well known to be fixed in the pupil plane. I.e. by rotating the individual frames before stacking in the image plane, quasi static speckles are getting azimuthally diluted. This is normally referred to a angular differential imaging $\left(\mathrm{ADI}^{15}\right)$. The spatial oversampling is highly beneficial for ADI analysis.

${ }^{*}$ The signal introduced by the thermal background stems from the cryostat entrance window, the telescope mirrors and molecular features from the atmosphere. This normally exceeds the peak flux in the PSF of even a bright star by $2-3$ orders of magnitude. 
For coronagraphy, to be placed in the image plane of VISIR, an Annular Groove Phase Mask (AGPM) is employed. At the time of writing this mask is being manufactured using an improved process compared to the device mounted in 2012. The AGPM is manufactured from a disc of synthetic diamond using complex lithography and reactive ion etching. The front-side carries a system of concentric rings with a sub-wavelength spacing. The Airy pattern of the star, if well centered will be rejected by this structure. Please note that Diamond has an index of 2.4 so that Fresnel losses without anti-reflective structure would be of order of $35 \%$. Hence two orthogonal grating structures are applied to the back. They form a system of sub-wavelength square frustra, which act as an effective medium, to provide for an anti-reflection coating. Outside of the coronagraphic spot, the device transmits $85 \%$. Further details of this, by now established type of coronagraph, can be found in literature ${ }^{8,9}$ and references therein).

The specific advantages of the AGPM are:

- relatively high attenuation ( $\geq 200$ in the first generation)

- as the attenuation needs full spatial coherence of the light the AGPM does not produce a black spot on the background signal but only a dip which is barely visible ${ }^{\dagger}$

- the inner working angle of the coronagraph is relatively small $(\leq \approx 200 \mathrm{mas})$

- the centre of the cold AGPM when viewed against the infrared dark sky, by scattering of ambient thermal radiation, produces a small but well visible dot of thermal signal which allows conveniently to measure the location of the occulting spot and hence facilitates all alignment

- by carefully tuning the geometry of the surface structure the device can be made to first order achromatic; we confirmed peak attenuation by a factor of $\approx 100$ for the astronomical $\mathrm{M}$ and Q-band $(\lambda \approx 5 \mu m$ and $\lambda \approx 20 \mu m$ respectively)

For the above reasons, the AGPM coronagraph is given preference over the 4-quadrant phase mask coronagraph $^{7}$ which would also be available for this experiment.

The beauty of the AGPM-coronagraph, its very small inner working angle, transforms itself into a serious operational handicap, that is to say in order to achieve in a real observation the contrast possible, a centering of the object "behind" the sweet spot of a fraction of the inner working angle is mandatory. In our case we are planning for a centering to typically \pm 10 mas. Meanwhile a variety of instruments have been equipped with AGPM masks so that a dedicated algorithm to correct the pointing as soon as the star becomes visible through the AGPM is available $\left(\mathrm{QACIT}^{16}\right)$.

\subsection{Details on the Lyot Stop}

Equally important to centering the star on the coronagraph is the proper positioning of the Lyot stop and apodizer. The very limited design space and the prohibitive effort to have a special insertion mechanism added to the VISIR camera forces us to mount the Lyot stop together with the filter. The stiffness and the high precision of repeatability of the VISIR filter wheel mechanism allows to reposition filter and Lyot stop with sufficient precision relative to the cold stop of the VISIR camera. The VISIR cold stop then is carefully aligned to the VLT-UT pupil, defined by M2, using the internal pupil imaging facility of VISIR.

Due to the need to employ rather complicated patterns (see fig. 1) there is no way to cut them from sheet metal. After a trade-off and some experiments it was decided to deposit the pattern directly to the filter substrate. The pattern is created by a deposit of chromium employing lithography and complicated wet chemical etching. Prototype activities have resulted in an approach where the chromium-mask is manufactured on an uncoated ZnSe substrate. Thereafter the coating manufacturer applies the filter coating, which however is opaque at

\footnotetext{
†A normal occulting coronagraph would produce a "black spot" of finite diameter where the background induced signal (see footnote on previous page) would drop to zero. Given the detector read-out circuitry such a feature would exceed the operational limits and produce, in the case of the VISIR detector, some 63 ghost images.
} 


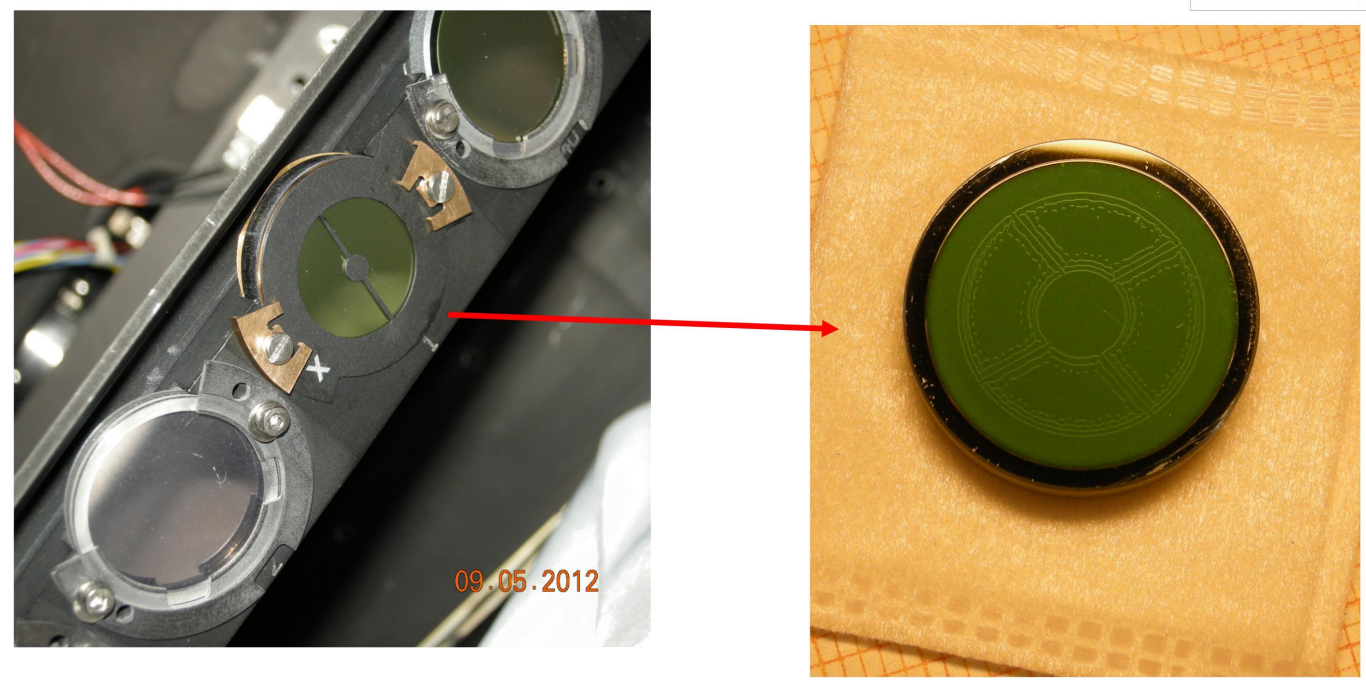

Figure 1. Left part shows the VISIR filter wheel with one of the Lyot stops cut into sheet metal and mounted together with the corresponding filter, state of the art in 2012. The right shows a prototype version of the second generation Lyot stop. Here the Lyot stop is substantially more refined (see text), deposited on the filter substrate and overcoated with the optical coating defining the interference filter. Even though the filter coating is opaque at visible wavelengths using dark field illumination the relief becomes clearly visible as the photo demonstrates. Thus this device lends itself for precision alignment of the mask to the instrument internal cold stop using standard alignment tools.

optical wavelengths. Still with dark field illumination the pattern relief becomes clearly visible, allowing for an rigorous precision alignment with a normal CCD-camera and the instrument open at room temperature.

The patterns employed in the pupil plane have a four fold function:

1. mask out the telescope spiders and central obscuration to minimize the thermal background radiation

2. mask out diffractive artifacts of the telescope spiders and central obscuration

3. provide for an apodized pupil stop

4. suppress the Airy rings of the second component of the double star (cf. fig 2).

\section{THE CHOPPER}

\subsection{Why Chopping}

As explained in some detail elsewhere ${ }^{17}$ signal modulation by chopping is required to allow for noise filtering. In a mid-IR imaging system the dominant noise sources are the background induced signal (white noise) and $1 / f$-noise resulting from instabilities of the camera in the presence of the very high background flux. In the case of VISIR being equipped with the Raytheon Aquarius detector there is additionally a noise component present resulting from the detector material referred to as Excess Low Frequency Noise, ELFN. ${ }^{18}$ For the case of VISIR with its specific illumination level and geometry, it was found that a chopping frequency of $3-5 \mathrm{~Hz}$ is mandatory to arrive at a meaningful rejection of ELFN, but typically $10 \mathrm{~Hz}$ would certainly yield an even better performance (cf. fig. 1 in this report ${ }^{18}$ suggests an improvement of $30 \%$ in signal to noise when increasing the chopping speed from 4 to $10 H z$.).

At VLT-UT3 classical M2 chopping with $\approx 4 H z$ is employed. With the move to UT4 maintaining M2chopping is not obvious. For the AOF the original VLT-M2 secondary and its large stroke tip-tilt actuators have been replaced with a thin shell mirror driven by voice coil actuators. So the standard way of chopping at the 

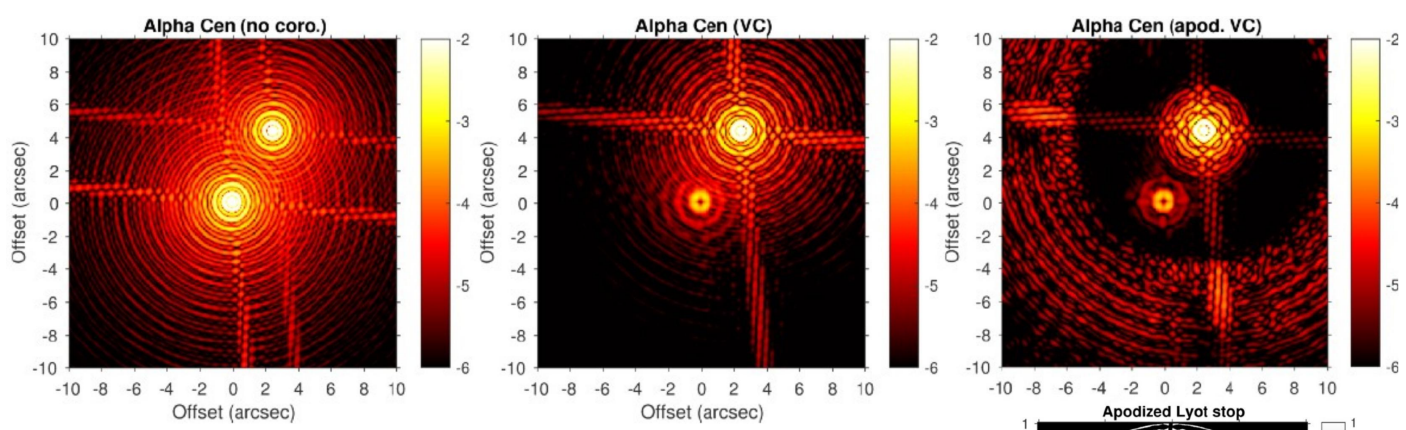

Simulations and design by G. Ruane (Caltech)

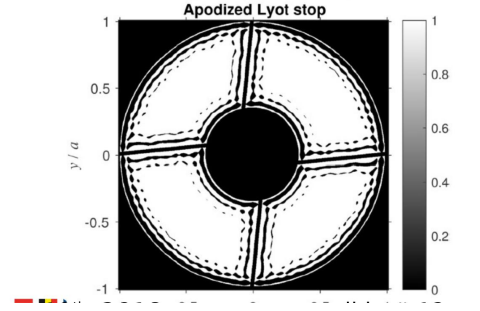

Figure 2. The left panel shows a simulated image of the two components of $\alpha$-Cen (logarithmic scale). The middle panel shows the expected exposure using the AGPM and a classical Lyot stop only. It can clearly be seen, that the Airy rings from the second component are the dominating background for the search for planets in the habitable zone around the occulted star. Hence a special apodizer is being added which suppresses the Airy rings for the relevant search volume. The right top image shows the resulting image for the mask, which by itself is shown in the lower right part.

VLT-UTs is not available at the AOF. Also the performance of the AO system with a chopped signal - hence a blinking wavefront reference star - was unpredictable.

Thus two avenues were followed: 1) the development of a focal plane chopper based on the pioneering concept of microwave radiometers ${ }^{19}$ and 2) the upgrade of the AOF-M2 operating software to enhance the tip tilt stroke of the M2 to allow for on sky chopping with a meaningful amplitude and duty cycle.

\subsection{The Dicke Switch}

The underlying principle here is to have a device which allows to observe the scientific field in one chopping cycle and to observe an artificial sky for the other chopping cycle. A regulated cryogenic black body is being tuned by a demon analyzing in real time the recorded frames in order to provide for exactly the same flux as that of the background radiation. The integrating sphere and the baffles are thermally insulated from the vacuum vessel and connected by copper straps to the cryogenic radiation shield of TIMMI2 in the lab and later to that of VISIR. Typical temperature is $\leq 130 \mathrm{~K}$. This technique of null-balancing had been applied in infrared astronomy previously highly successfully for Solar mid-infrared spectroscopy. ${ }^{20,21}$

Beyond this, the vacuum vessel housing the Dicke switch also provides for a convenient way of splitting optical light for wave front sensing from the IR-signal. A ZnSe window with a custom made coating is being used featuring $97 \%$ transmission for the filter bandpass used for the NEAR campaign. The rotating D-mirror is controlled via a commercial vacuum compatible angle gear using helical gearing to minimize vibrations. The assembly is powered by a DC motor outside of the cryostat vessel employing a ferrofluidic feedthrough to enter the cryostat vacuum. The D-mirror is dynamically balanced and provides for chopping frequencies up to $10 \mathrm{~Hz}$. There are sync signals to allow for proper phasing of the detector read out.

To enable the extension of the vacuum vessel and to create space for the wavefront sensor and camera, the original calibration unit of VISIR is being re-arranged.

The Dicke switch is presently being tested in the laboratory in Garching. Sky and telescope are being simulated the "standard" laboratory way by using a $L N_{2}$ cooled load which is imaged with a Germanium lens acting as telescope through a set of thin plastic foils to achieve the proper emissivity of the VLT in normal operation. 


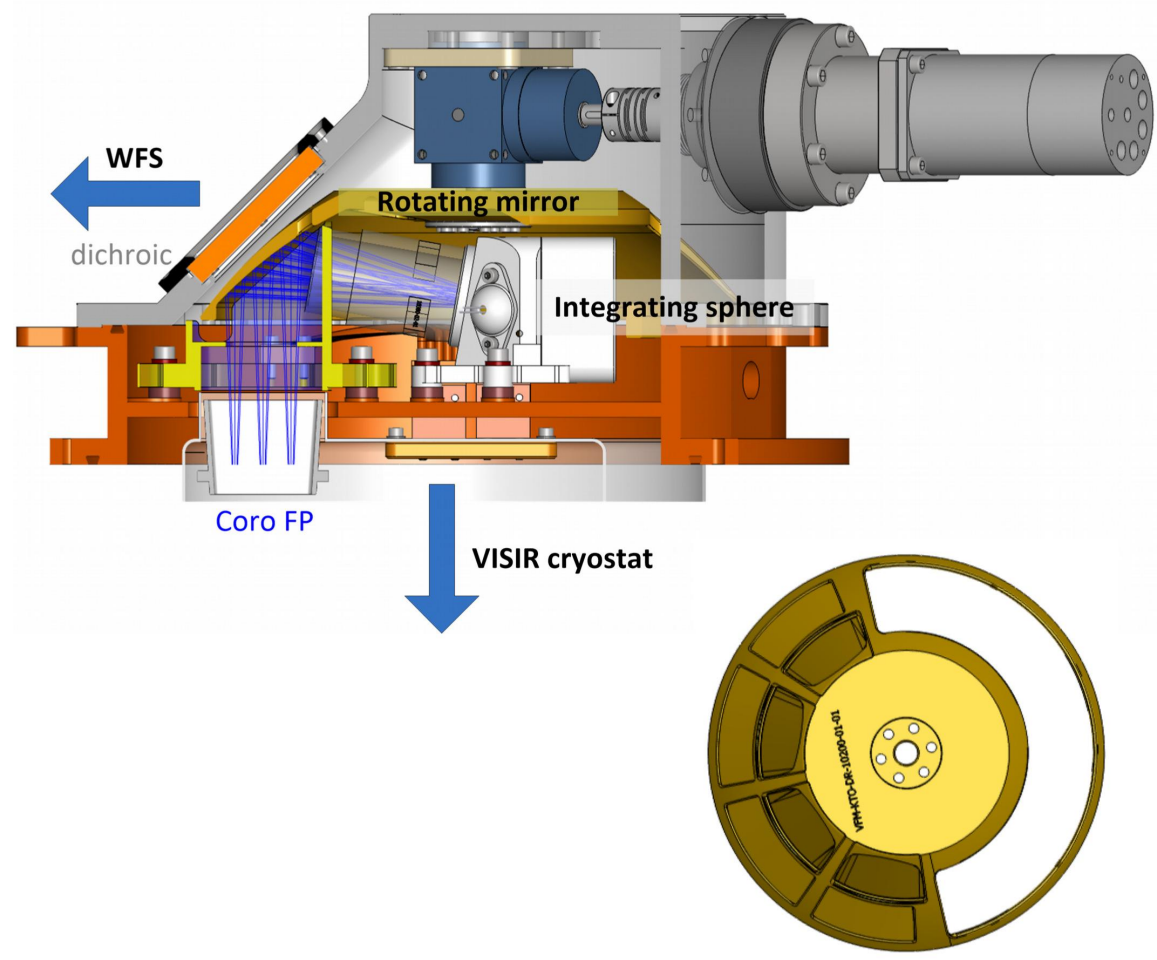

Figure 3. Cut drawing of the new VISIR internal chopper based on the then revolutionary concept of microwave pioneer Robert Dicke of 1946. The unit will be mounted to the VISIR cryostat as an extension in the direction of the telescope. It comprises of a toroidal rotating D-mirror which allows the detector to observe alternatingly the sky, or a cryogenic integrating sphere with a regulated black body. The unit also carries a dichroic which provides for the pick-off of the light for the AO wave front sensor (design Kampf Telescope Optics, KTO).

\subsection{Chopping with the Adaptive M2}

Parallel to the Dicke switch the supplier of the AOF-M2 was contacted. Eventually, a change of the operations firmware was made. Now there is sufficient stroke in the actuators to allow for chopping with an amplitude of $\approx 5$ " projected on sky. The changed configuration has been successfully tested on sky observing a double star (HIP 48131, separation 4.3"). Even at frequencies as high a $10 \mathrm{~Hz}$ the settling time is short enough to allow for a duty cycle approaching $90 \%$.

In case of $\alpha$-Cen, it is possible, and planned, to chop between the two components of the system so that one can search two stars for planets for the price of one.

\subsection{Conclusions on Chopping}

In both cases, AOF-M2-chopping and the Dicke switch, the goal was to enable chopping with frequencies as high as $10 \mathrm{~Hz}$ to be really in a scheme where the ELFN noise can be rejected to a higher degree. This has been achieved. Hence the ELFN noise contribution will be less than that for the test case discussed above, where data were taken with $f_{\text {chopp }}=4 H z$. So indeed one can expect the noise to drop by some $30 \%$ compared to the estimate given in chapter 2 .

At time of writing we are lacking a noise measurement in the laboratory with sky simulation to confirm that the Dicke switch will deliver the same noise as the M2 chopping.

After the NEAR campaign the Dicke switch will be retrofitted with the original entrance window of VISIR (WFS camera and optics removed). The Dicke switch may be highly valuable for standard observations of extended objects. M2 chopping, on average, is highly compromised in case of extended objects or objects in 


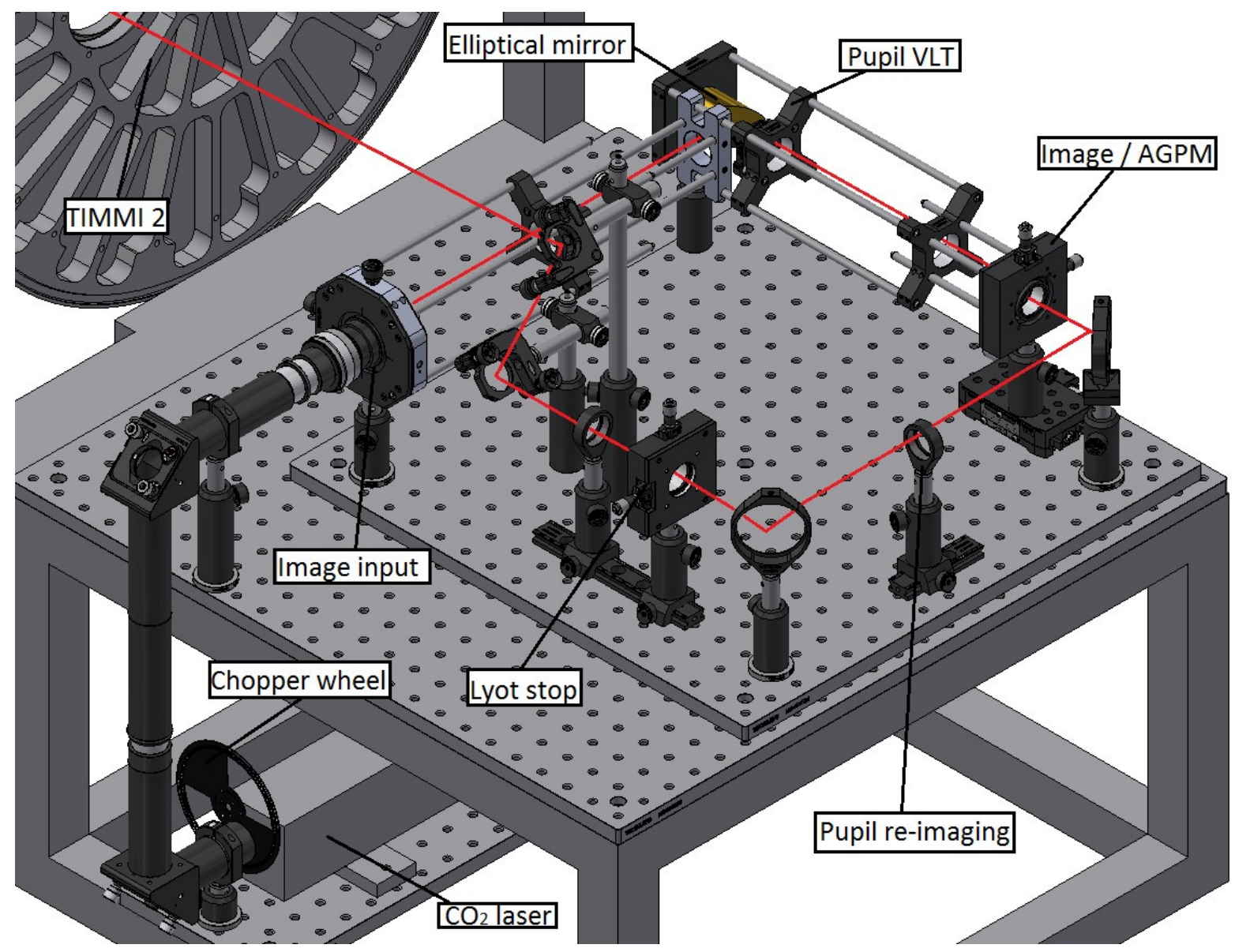

Figure 4. The design sketch shows the optical bench for testing the next generation AGPMs. The optical path is highlighted in red. As we expect intrinsic extinction ratios well above $10^{-3}$ such tests cannot be done with a classical black body for lack of intensity. Therefore a line tunable $\mathrm{CO}_{2}$-laser has been procured. This laser is attenuated by a defocused spatial filter labeled image input. The elliptical mirror under $90 \mathrm{deg}$ will focus the light in the plane image/AGPM. Thereafter a Ge-lens forms a pupil of the size of the VISIR cold stop. Here apodizers, the Lyot stop and any other parts potentially foreseen to be inserted in the VISIR pupil plane can be qualified. As can be seen, the elements around the elliptical mirror are a sub-assembly on an extra optical bench which can be dismounted. The alignment of the elliptical mirror thus can be tested separately with a standard shop interferometer and a HeNe-laser to ensure that the optical quality of the beam at the AGPM is sufficient for a meaningful test. As an alternative, at the location of Image/AGPM the ZELDA mask can be placed. In combination with an extra Ge-lens TIMMI2 can be operated in pupil imaging mode which would provide for a second independent rigorous test of the proper alignment of the elliptical mirror. 
associations where simply no nearby empty reference fields exist. So the Dicke switch will boost the use of VISIR also for normal observations.

\section{LAB TESTING OF APODIZERS, NEW AGPM ETC.}

Figure 4 shows the set-up for performing tests of all components to be mounted within VISIR. Rather than mounting the AGPM to the TIMMI2 aperture wheel this approach has been chosen as it allows for a rigorous analysis of the optical quality of the light at the AGPM test location. Moreover, any interference by potential vibrations or differential movements of the "calibration star" on the warm bench outside of the TIMMI2 vessel relative to the cold optical bench can be avoided.

In addition the ZELDA ${ }^{22}$ mask (see below) will be brought to the AGPM location as well. This allows to test the mask and the algorithms as much as to use the ZELDA technique for an independent assessment of image quality in the AGPM test.

The tests will begin as soon as the Dicke switch is qualified.

\section{OTHER TECHNICALITIES}

As pointed out above, a WFS camera (ESO spare part) plus a special adaptive optics train is being integrated into the adaptor flange connecting the VISIR vessel to the telescope instrument rotator flange. To that end the old calibration unit (IR-source, chopper, monochromator, pinhole and Offner projection system into the focal plane) will be reconfigured. The Offner relay will be replaced by an off-axis elliptical mirror. This mirror will be mounted directly to the monochromator and moved together with the pinhole. In this way it becomes possible to scan the full field with the calibration source, in spite of the small aberration free field of the elliptical mirror.

In the VISIR focal plane, the ZELDA ${ }^{22}$ mask will be installed on the mask wheel which can be optionally inserted instead of the field stop or the AGPM mask. Together with the VISIR pupil imaging this mask allows to determine and quantify residual wavefront errors due to e.g. misalignment of the elliptical mirror in the calib. unit, or due to non-common path aberrations in case of AO operations.

In principle, there would be a need to have an atmospheric dispersion compensator in the AO-WFS optical path. This extra effort is avoided by using a narrowband filter around $900 \mathrm{~nm}$ for the wave front sensing. For a fast track experiment, the loss in light appears tolerable for bright stars compared to the extra complication of having a compensator.

VISIR needs a major cable bundle, 8 semi-rigid He-pressure lines plus cooling water. In order to mount VISIR to UT4 Cassegrain with minimum interference and cost, we will resort to the solution which was the baseline how to mount VISIR at the time of the Final Design Review (1999). It will basically be a pre-balanced chain hanging from Cassegrain. The approach to pre-balance is limiting the extra torque on the altitude axis to $\pm 400 \mathrm{~N}$ depending on Zenith distance. This extra torque should not pose any problem for the telescope altitude motor and its control loop.

\section{CONCLUSIONS}

VISIR shall be equipped with the most advanced AGPM Lyot coronagraph in combination with a sophisticated apodizer mask. This instrument will then be brought to the Cassegrain focus of ESO-UT4 now featuring an adaptive secondary.

Preparatory laboratory tests will be finalized in Oct. 2018. In Dec. 2018 VISIR will be removed from UT3 for the intervention. A first installation of VISIR on UT4 is planned for the first quarter of 2019 for commissioning. Finally, anticipating a successful commissioning, in late spring / early summer the $100 h$ observing campaign for both components of $\alpha$-Cen will take place.

Data will be analyzed by us in real time using established recipes. As the observation is done in director's discretionary time (DDT) all data also become available to the community immediately for independent analysis.

All technical expertise gained here will be shared with the team for the thermal infrared instrument, METIS, ${ }^{23}$ one of the first light instruments of ESO's E-ELT. 
Last not least, when VISIR will return to UT3 the Dicke switch will provide for an alternative to M2 chopping which certainly will be highly valuable for standard observations of extended objects or crowded fields.

\section{ACKNOWLEDGMENTS}

This project was only possible with the enthusiastic support of many staff at ESO, but also in the community. Of particular importance is a grant by the Breakthrough Initiatives and the generous offer of DDT by ESO's Director General.

\section{REFERENCES}

[1] A.F.M. Moorwood, 1982, Infrared Instrumentation at ESO, The Messenger, 27, p. 11

[2] H.-G., Reimann et al., 2000, TIMMI2: a new multimode mid-infrared instrument for the ESO 3.6-m telescope, Proc. SPIE Vol. 4008, pp. 1132-1143

[3] H.U. Käufl et al. 2003 TIMMI2 ESO's Thermal Infrared Multimode Instrument: Technical Description and Some Scientific Results, Proceedings of the SPIE, Volume 4841, pp. 117-128

[4] P.-O. Lagage et al. 2004, Successful Commissioning of VISIR: The Mid-Infrared VLT Instrument, The Messenger 117, p. 12-16

[5] H.U. Käufl et al., 2015, The Return of the Mid-infrared to the VLT: News from the VISIR Upgrade, The Messenger159, p. 15-18

[6] F. Kerber et al., 2016, VISIR upgrade overview: all's well that ends well, Proceedings of the SPIE, Volume 9908 , pp. 13

[7] D. Rouan et al., 2000, The Four-Quadrant Phase-Mask Coronagraph. I. Principle, PASP 112, Number 777

[8] D. Mawet et al., 2005, Annular Groove Phase Mask Coronagraph, ApJ, 633, pp. 1191-1200

[9] O. Absil et al., 2018, Around the world: status and prospects with the infrared vortex coronagraph, Proceedings of SPIE Conference Ground-based and Airborne Instrumentation for Astronomy VII, paper 10702-29, in press

[10] C. Delacroix et al., 2012, A diamond AGPM coronagraph for VISIR, Proceedings of the SPIE, Volume 8446, article id. $84468 \mathrm{~K}, 9$ pp.

[11] D. Asmus et al., 2016, Science Verification for the VISIR Upgrade, The Messenger, 164, p. 14-16

[12] P.-Y. Madec et al., 2018, Adaptive Optics Facility: from an amazing present to a brilliant future, Proceedings of SPIE Vol. 10703 Adaptive Optics Systems VI, paper 10703-2

[13] H.U. Käufl et al., 2000, From ISAAC to GOLIATH, or better not!? Infrared instrumentation concepts for $100 \mathrm{~m}$ class projects, Proceedings of the Backaskog workshop on extremely large telescopes : Backaskog Castle, Sweden, June 1-2, 1999, ESO conference and workshop proceedings, no. 57, p.282

[14] R. Siebenmorgen et al., 2004, Mid-infrared emission of galactic nuclei, A\&A 414, 123-139

[15] C. Marois et al. 2006, Angular Differential Imaging: A Powerful High-Contrast Imaging Technique, ApJ 641 P. 556

[16] E. Huby et al., 2017, On-sky performance of the QACITS pointing control technique with the Keck/NIRC2 vortex coronagraph, A\&A 600, A46

[17] H.U. Käufl et al., 1991 A sky-noise measurement and its implication for ground-based infrared astronomy in the 10-micron atmospheric window, ExpAst 2 p.115

[18] D. Ives et al., 2014 AQUARIUS: the next generation mid-IR detector for ground-based astronomy, an update, Proceedings of the SPIE, Volume 9154, id. 91541J 11 pp.

[19] R.H. Dicke, 1946, The Measurement of Thermal Radiation at Microwave Frequencies, Review of Scientific Instruments, 17 Issue 7, p.268-275

[20] D. Deming et al., 1988, Infrared Helioseismology - Detection of the Chromospheric Mode, IAU Symposium, No. 123. p. 425

[21] D.A. Glenar et al., 1988, Infrared Heterodyne Spectroscopy - a Tool for Helioseismology, IAU Symposium, No. 123. p. 425

[22] N'Diaye, 2013, Calibration of quasi-static aberrations in exoplanet direct-imaging instruments with a Zernike phase-mask sensor, A\&A, 555 id.A94, 11 pp.

[23] B. Brandl et al., 2018, Status of the mid-infrared ELT imager and spectrograph METIS, Proceedings of SPIE Conference Ground-based and Airborne Instrumentation for Astronomy VII, paper 10702-66, in press 